Chang Xu

Key Laboratory for Mechanics in

Fluid Solid Coupling Systems, Institute of Mechanics,

Chinese Academy of Sciences,

No. 15 Beisihuanxi Road,

Beijing 100190, China;

School of Engineering Science, University of Chinese Academy of Sciences,

No. 19(A) Yuquan Road,

Shijingshan District,

Beijing 100049, China

Yiwei Wang ${ }^{1}$

Key Laboratory for Mechanics in

Fluid Solid Coupling Systems, Institute of Mechanics,

Chinese Academy of Sciences,

No. 15 Beisihuanxi Road,

Beijing 100190, China;

School of Engineering Science,

University of Chinese Academy of Sciences,

No. 19(A) Yuquan Road,

Shijingshan District,

Beijing 100049, China

e-mail: wangyw@imech.ac.cn

\title{
Chenguang Huang
}

Key Laboratory for Mechanics in

Fluid Solid Coupling Systems, Institute of Mechanics,

Chinese Academy of Sciences,

No. 15 Beisihuanxi Road,

Beijing 100190, China;

School of Engineering Science,

University of Chinese Academy of Sciences,

No. 19(A) Yuquan Road

Shijingshan District,

Beijing 100049, China

Chao Yu

Key Laboratory for Mechanics in

Fluid Solid Coupling Systems, Institute of Mechanics,

Chinese Academy of Sciences,

No. 15 Beisihuanxi Road,

Beijing 100190, China

School of Engineering Science,

University of Chinese Academy of Sciences,

No. 19(A) Yuquan Road,

Shijingshan District

Beijing 100049, China

Jian Huang

Key Laboratory for Mechanics in

Fluid Solid Coupling Systems, Institute of Mechanics,

Chinese Academy of Sciences,

No. 15 Beisihuanxi Road,

Beijing 100190, China;

School of Engineering Science, University of Chinese Academy of Sciences,

No. 19(A) Yuquan Road, Shijingshan District,

Beijing 100049, China

\section{Cloud Cavitating Flow That Surrounds a Vertical Hydrofoil Near the Free Surface}

Unstable cavitation presents an important speed barrier for underwater vehicles such as hydrofoil craft. In this paper, the authors concern about the physical problem about the cloud cavitating flow that surrounds an underwater-launched hydrofoil near the free surface at relatively high-Froude number, which has not been discussed in the previous research. A water tank experiment and computational fluid dynamics (CFD) simulation are conducted in this paper. The results agree well with each other. The cavity evolution process in the experiment involves three stages, namely, cavity growth, shedding, and collapse. Numerical methods adopt large eddy simulation (LES) with Cartesian cut-cell mesh. Given that the speed of the model changes during the experiment, this paper examines cases with varying constant speeds. The free surface effects on the cavity, re-entry jet location, and vortex structures are analyzed based on the numerical results.

[DOI: $10.1115 / 1.4036669]$

${ }^{1}$ Present address: Room 201, Building 2, No. 15 Beisihuanxi Road, Beijing 100190 , China.

Contributed by the Fluids Engineering Division of ASME for publication in the JouRnal OF FluidS EngineERING. Manuscript received December 15, 2016; final manuscript received April 12, 2017; published online July 10, 2017. Assoc. Editor: Hui Hu. 


\section{Introduction}

Cavitation often occurs during a liquid-gas phase transfer that is driven by the decreased local pressure at the surface of high-speed underwater vehicles. The formation, development, and collapse of cavitation induce erosion, noise, and vibration. Cavitation has always been in the forefront of the research $[1,2]$. The influence of cavitating flow on the performance of navigation vehicles cannot be neglected, and the pressure pulsation, vibration, and ballistic change induced by an unsteady cavitation evolution present a great concern. Cavitating flow is often examined through a water tunnel [3] or water tank test [4]. Computational fluid dynamics (CFD), which has been integrated into commercial software FLUENT and CFX [5-7] as well as into open-source [8-11] and in-house [12-16] software, has been recently introduced as a major approach for investigating cavitation. The cavitating flow of the unsteady cloud around the foil [17-19], propeller models [20], and other underwater vehicles $[7,21]$ presents typical problems.

Vertical hydrofoil is a typical support structure for the highspeed craft $[22,23]$ near the free surface. However, the interaction between the free surface and cavitation presents a very complex and interesting problem for the surface and near-surface highspeed craft. Faltingsen proposed a numerical method for calculating free surface and steady super cavity based on the potential flow theory and analyzed the important parameters that influence the cavity shape development [24,25]. Bals and Kinnas used the boundary element method to investigate the cavitation problems of submerged and surface piercing hydrofoils as well as to validate his experimental results [26-28]. Wang et al. conducted an experiment and simulation to examine the characteristics of unsteady cloud cavitation evolution on the surface of a projectile, and then analyzed the interaction between the free surface and cavitation [29]. Gnanaskandan and Mahesh employed two simulation methods, namely, large eddy simulation (LES) and a self-developed predictor-corrector approach, to simulate the cloud cavitation around a wedge [30,31]. Young and coworkers examined the ventilated cavities on a surface piercing hydrofoil at moderate Froude numbers $[32,33]$. The formation and elimination mechanisms are proposed and stability regions of the different flow regimes are plotted. Besides, the authors also give a comprehensive review of scaling effect on the ventilation of lifting bodies. However, only few studies have examined the unsteady cloud cavitation, thereby, leaving many other problems to be solved in future research.

Vehicles often change their speed during their operation. Studying the effects of changing velocity on cavitation requires complex simulation and test equipment, thereby hindering further research on such effects. The changes in the natural supercavitating flow under deceleration conditions have been analyzed using the numerical method [34]. Shin et al. [35] applied the numerical method to simulate an unsteady cavitating flow through a two-dimensional decelerating cascade. Chen et al. [36] investigated the collapse regimes of the cavitation on submerged vehicles under deceleration conditions. Various turbulent models have also been proposed. Wang et al. [37] conducted an experiment and numerical analysis on the internal cavitation bubbles collapse of a projectile that is vertically launched underwater during the deceleration process.

Some new phenomena are revealed in this paper. To examine the cloud cavitating flow that surrounds a vertical hydrofoil near the free surface, this research is divided into two parts, namely, the water tank launch experiment discussed in Sec. 2 and the CFD simulation in Sec. 3. The numerical and test results agree well with each other. Detailed mechanisms inside the cavitating flow can be analyzed through the simulation results in Sec. 4. Simulation of various constant speed cases are also discussed in Sec. 4.1 due to the deceleration of the hydrofoil during the water tank experiment. Section 5 concludes the paper.

\section{Water Tank Experiment}

2.1 Description of Test Facility. The vertical hydrofoil model used in the experiment comprises aluminum, and the front end of the model is painted black to facilitate observation. The cross-sectional shape of the model is a rectangular isosceles right

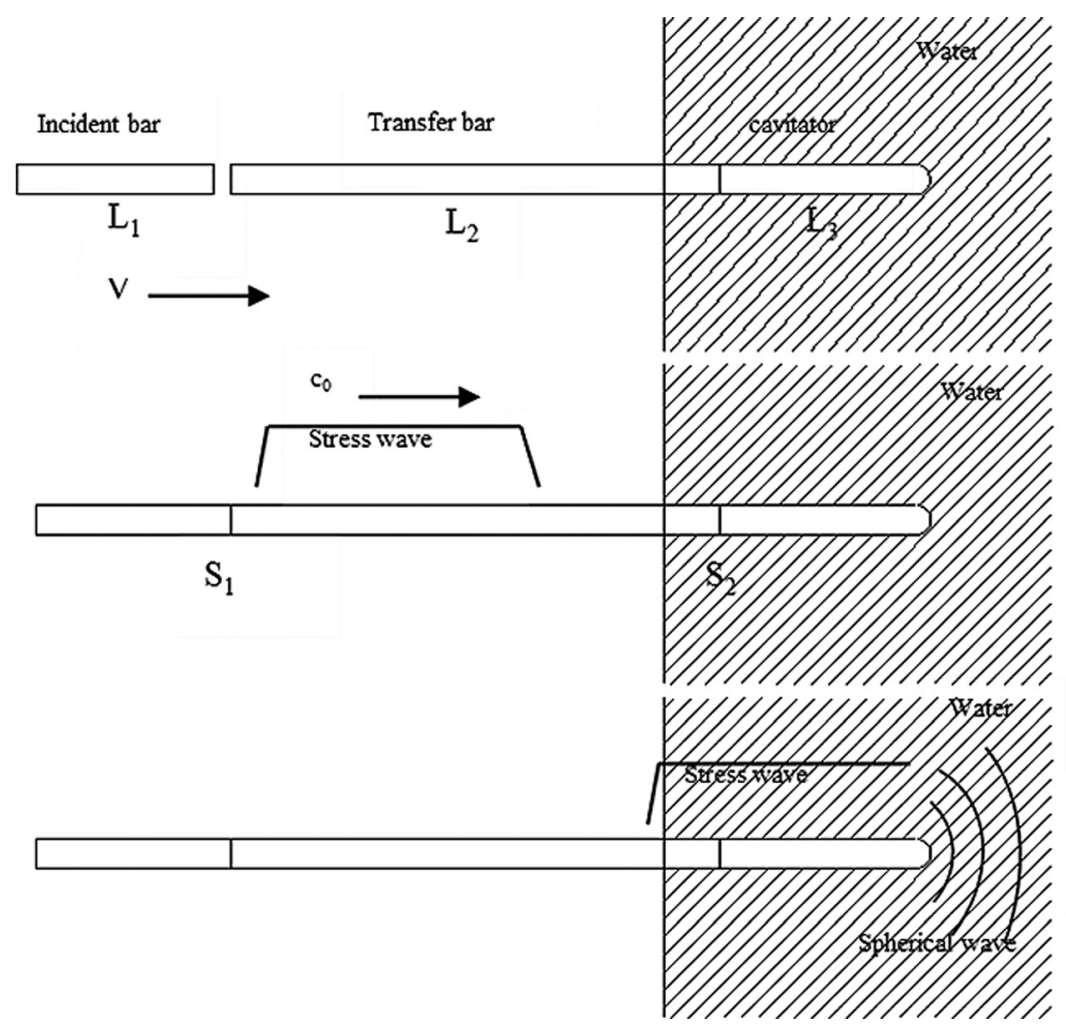

(a)

Fig. 1 The Split-Hopkinson pressure bar technology. Three parts the incident bar, the transfer bar, and the test model are included. The launch process is shown. 


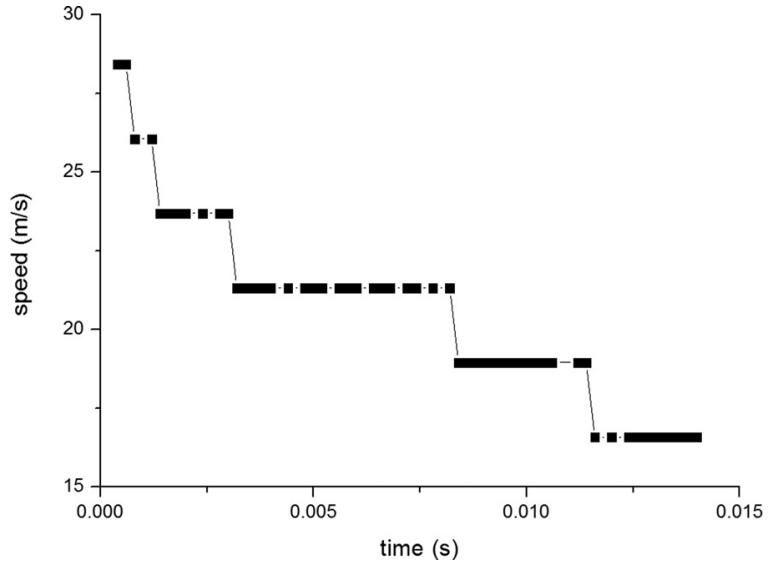

Fig. 2 Speed of the tested hydrofoil changes with time in the water tank experiment from $t=0.001 \mathrm{~s}$ to $t=0.014 \mathrm{~s}$

triangle. The model dimensions are $120 \mathrm{~mm} \times 20 \mathrm{~mm} \times 100 \mathrm{~mm}$, and the upper side of the model is located $20 \mathrm{~mm}$ away from the free surface. Figure 1 shows the schematic diagram of the Split-Hopkinson pressure bar technology [4] used in the experiment as a launching source under typical conditions. Initially, the model is instantly accelerated to about $20 \mathrm{~m} / \mathrm{s}$ in $200 \mu \mathrm{s}$ and then launched into a $1 \mathrm{~m} \times 1 \mathrm{~m} \times 2 \mathrm{~m}$ water tank. The water temperature inside the tank is about $20^{\circ} \mathrm{C}$. The entire experiment is recorded using a high-speed camera with a sampling frequency of 12,000 frames per second.

The model speed can be derived from the changes in the leading edge location of the model as seen in the adjacent images, which varies from about $26 \mathrm{~m} / \mathrm{s}$ to $16 \mathrm{~m} / \mathrm{s}$. Figure 2 shows that the model speed changes in the first $0.014 \mathrm{~s}$.

2.2 Typical Experiment Results and Analysis. Resistance has a non-negligible effect on the speed of the launched model. The launching speed of the model at the beginning and end of the test can be derived from changes in the leading edge location of the model as can be seen in the adjacent images, which varies from $26 \mathrm{~m} / \mathrm{s}$ to $16 \mathrm{~m} / \mathrm{s}$. The cavitation number can be calculated as follows:

$$
\sigma=\frac{p_{\infty}-p_{v}}{\frac{1}{2} \rho_{l} v_{\infty}^{2}}=0.49
$$

where $p_{\infty}$ is the standard atmospheric pressure, $p_{v}$ is the saturated vapor pressure, $\rho_{l}$ is the liquid water density, and $v_{\infty}$ is the launch speed. Given the small size and high speed of the model, the

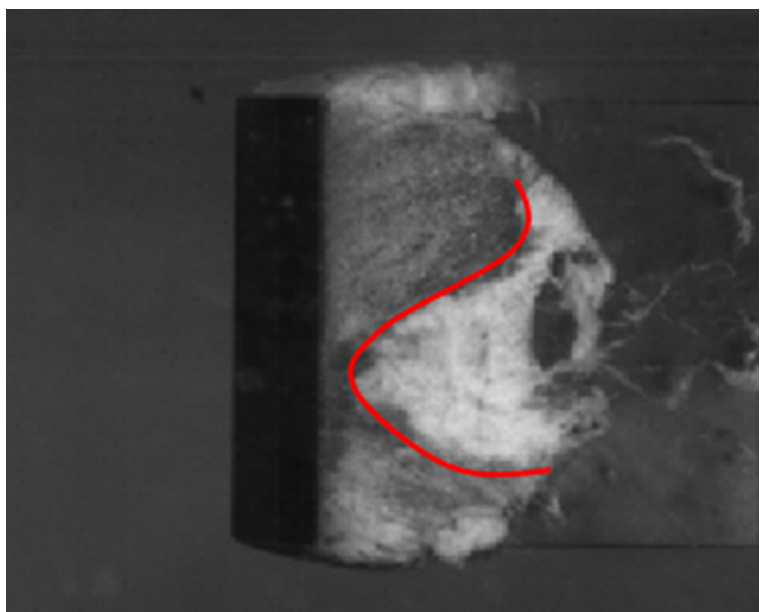

Fig. 3 Typical cavitation at $t=0.006 \mathrm{~s}$. The white foam like reentry jet inside cavity is marked by the line.

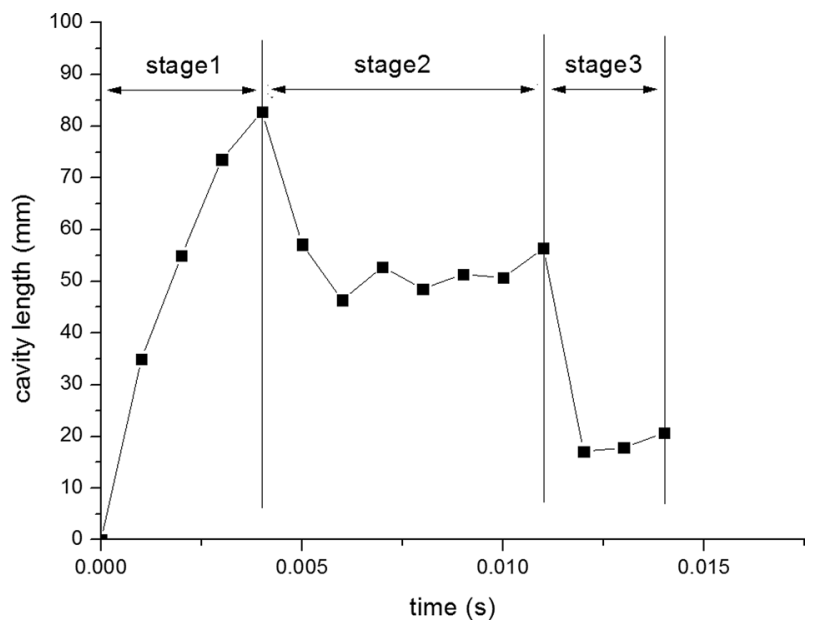

Fig. 4 Cavity length changes with time from $t=0 \mathrm{~s}$ to $t=0.014$ s. Three stages of the cavity evolution are marked.

difference between the pressure exerted by gravity at the upper and lower sides of the model is lower than the flow dynamic pressure. The equation $\left(\rho_{l} g d /(1 / 2) \rho_{l} v_{\infty}^{2}\right)=0.0018 \ll 1$ suggests that the changes in cavitation number in the $y$ direction can be ignored, where $d=37 \mathrm{~mm}$ represents the projectile diameter.

Figure 3 shows a typical cavitation image taken at $t=0.006 \mathrm{~s}$. The cavitating flow around the hydrofoil is in the development stage. The line in the figure indicates the profile of the re-entry jet front. The re-entry jet inside the cavity moves toward the leading edge of the cavity. In the experiment, the re-entry jet is deflected from the free surface under the free surface effect. From the figure, we can measure the cavity length and position of the re-entry jet as well as observe the cavity evolution process. The precision of the length and thickness is approximately a pixel of the image, which stands for $0.671 \mathrm{~mm}$. The experimental results can be used to validate the accuracy of the numerical simulation method.

2.3 Cavity Evolution Process. The cavity evolution process involves three stages. Figure 4 shows how the cavity length changes with time in the first $0.014 \mathrm{~s}$. The cavitating flow increases at the first stage. The total cavity length increases along with the decreasing growth rate before reaching its peak. The re-entry jet inside the cavitating flow moves toward the leading edge of the model and eventually cuts off the cavity by interfering with the main flow, thereby leading to a sharp contraction in the cavity length. The cavity shape eventually reaches a relatively stable state. The cavity collapses in the last stage, and then, the cavity length drastically decreases. The re-entry jet inside the cavitating flow exerts a non-negligible influence on cavity evolution. Figures 5-7 show the typical cavitation in the three aforementioned stages. This study focuses on the sheet and cloud cavities at the surface of the hydrofoil than that at the upper and lower sides of the model.

Figure 5 shows the growth of the cavity and the movement of the re-entry jet toward the leading edge of the hydrofoil. The two typical moments of $t=0.002 \mathrm{~s}$ and $t=0.006 \mathrm{~s}$ are compared and analyzed. By comparing the length of the cavity on the upper and lower sides of the model, we can see that the length of the cavity on the upper side of the hydrofoil is slightly shorter than that of the cavity on the lower side under the free surface effect. Free surface also greatly affects the re-entry jet inside the cavity. The re-entry jet does not move toward the center of the entire cavity at $t=0.006 \mathrm{~s}$, but is deflected from and is located parallel to the free surface.

Cavity shedding occurs when the re-entry jet reaches the leading edge of the hydrofoil. Figure 6 shows the experimental results of the cavitation in the contraction phase at $t=0.008,0.009,0.01$, and $0.011 \mathrm{~s}$. At $t=0.008-0.011 \mathrm{~s}$, the re-entry jet inside the cavity 

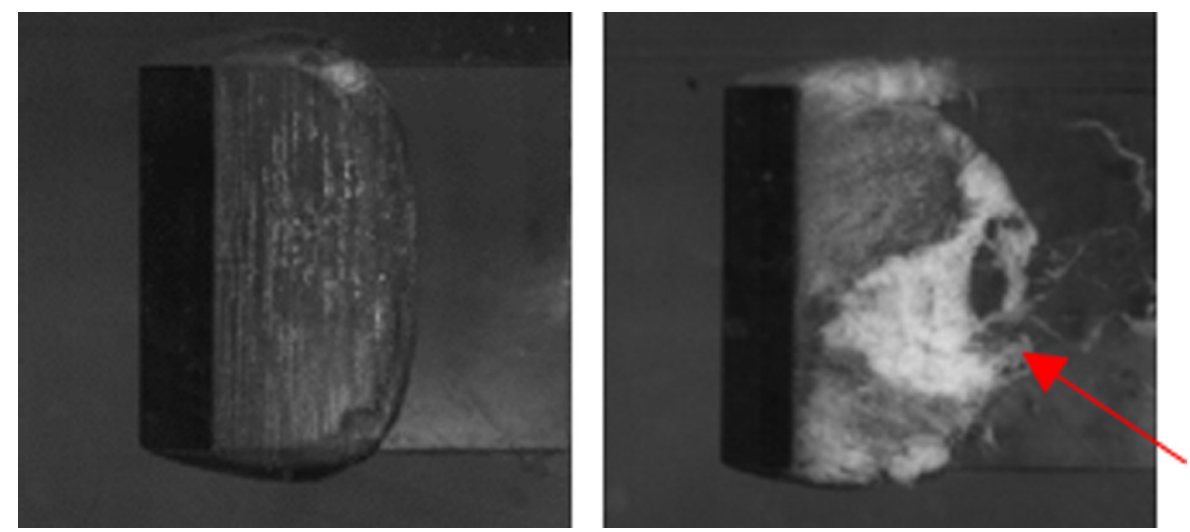

Fig. 5 Typical cavitation in cavity growth stage $1(0.002,0.006 \mathrm{~s})$. The re-entry jet inside the cavity which is deflected from and is located parallel to the free surface is pointed out by the arrow.
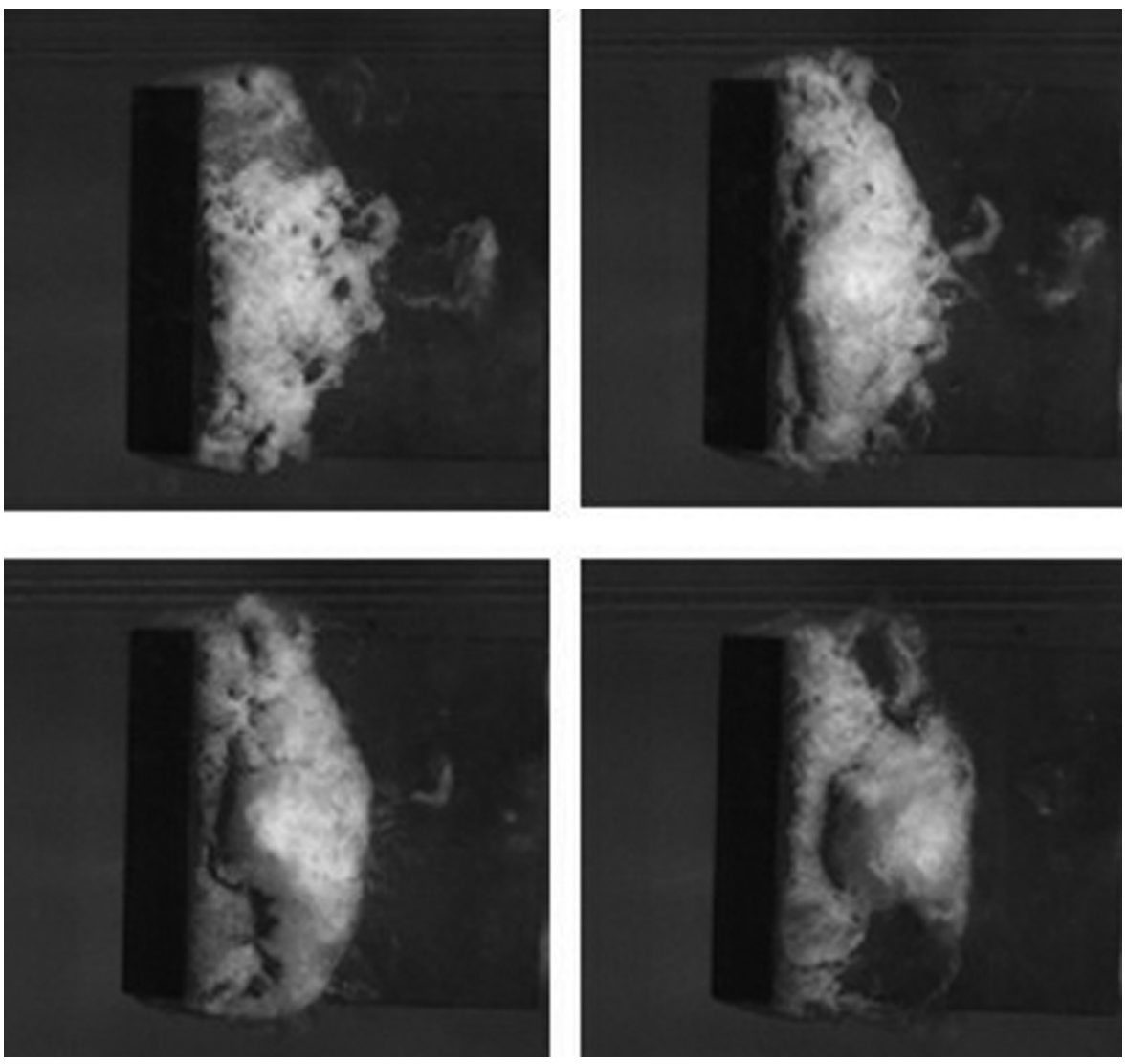

Fig. 6 Typical cavitation in cavity shedding stage $2(0.008 \mathrm{~s}, 0.009 \mathrm{~s}, 0.01 \mathrm{~s}$, and $0.011 \mathrm{~s})$

reaches the leading edge of the cavity, cuts off the whole cavity by interfering with the main flow, and then propagates up and down to induce cavity shedding. The shedding cavity gradually shrinks to the middle and breaks away from the main cavity. During this process, the overall shape of the cavity remains stable, while the cavity length slightly changes. The stability of the whole cavitating flow reaches a critical state at $t=0.011 \mathrm{~s}$.

Figure 7 shows the two typical moments of $t=0.013 \mathrm{~s}$ and $0.014 \mathrm{~s}$ during the cavity collapsing stage. The cavity length decreases more sharply at this stage than in the previous stage. The cavity in the previous stage collapses, and a new cavity is generated in the following stage. The total cavity length slightly increases during this process. Figure 7 also shows the horseshoe cavity structure induced by the re-entry jet.

\section{Numerical Method}

3.1 Governing Equations. Navier-Stokes equations about single fluid/multiple components are widely used to solve the liquid/gas two phases flow problems. The continuity and momentum equations are

$$
\begin{gathered}
\frac{\partial \rho}{\partial t}+\frac{\partial\left(\rho u_{j}\right)}{\partial x_{j}}=0 \\
\frac{\partial\left(\rho u_{i}\right)}{\partial t}+\frac{\partial\left(\rho u_{i} u_{j}\right)}{\partial x_{j}}=-\frac{\partial p}{\partial x_{j}}+\frac{\partial}{\partial x_{j}}\left(\mu \frac{\partial u_{j}}{\partial x_{j}}\right)
\end{gathered}
$$

where $u_{i}$ is the velocity component in $i$ direction, $\rho$ is the mixture density, $p$ is the pressure, and $\mu$ is the laminar viscosity which can be defined as 

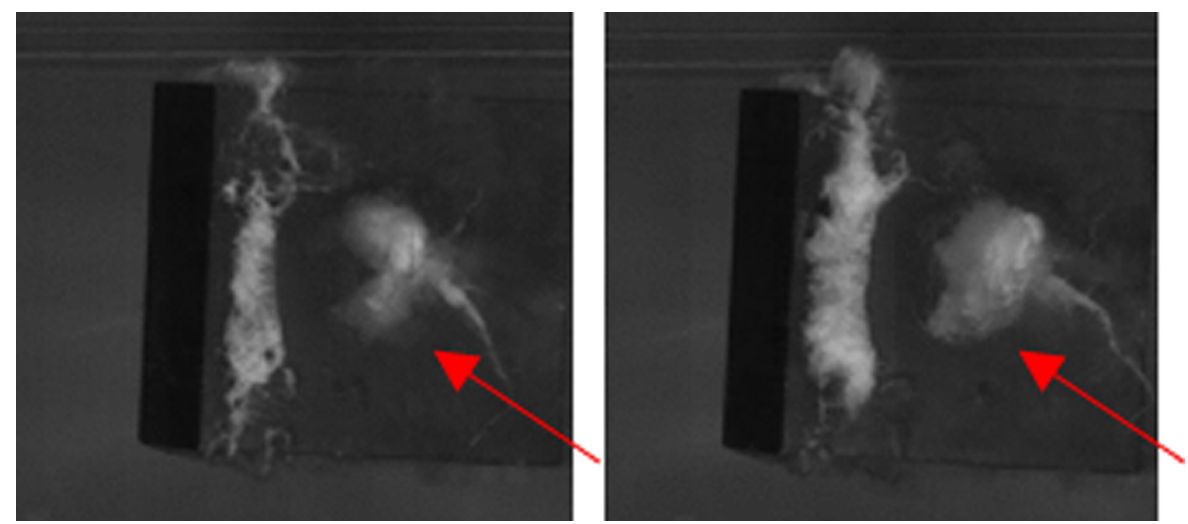

Fig. 7 Typical cavitation in cavity collapsing stage $3(0.013 \mathrm{~s}$ and $0.014 \mathrm{~s})$. The horseshoe cavity structure induced by the re-entry jet is pointed out by the arrows.

$$
\mu=\left(1-\alpha_{v}\right) \mu_{l}+\alpha_{v} \mu_{v}
$$

where $\alpha$ is the volume fraction of the different phases, and $l$ and $v$ represent liquid water and water vapor, respectively. The mixture density $\rho$ is defined as

$$
\rho=\left(1-\alpha_{v}\right) \rho_{l}+\alpha_{v} \rho_{v}
$$

The transport equation of the vapor volume fraction is

$$
\frac{\partial\left(\alpha_{v} \rho_{v}\right)}{\partial t}+\frac{\partial\left(\alpha_{v} \rho_{v} u_{j}\right)}{\partial x_{j}}=\dot{m}^{+}-\dot{m}^{-}
$$

where $\dot{m}^{+}$and $\dot{m}^{-}$are the mass transfer rate of evaporation and condensation, derived from the Rayleigh-Plesset bubble dynamics equations by Zwart et al. [38]

$$
\begin{gathered}
\dot{m}^{+}=F_{\text {vap }} \frac{3 a_{\text {nuc }}\left(1-\alpha_{v}\right) \rho_{v}}{R_{B}} \sqrt{\frac{2}{3} \frac{\max \left(p_{v}-p, 0\right)}{\rho_{l}}} \\
\dot{m}^{-}=F_{\text {cond }} \frac{3 \alpha_{v} \rho_{v}}{R_{B}} \sqrt{\frac{2}{3} \frac{\max \left(p-p_{v}, 0\right)}{\rho_{l}}}
\end{gathered}
$$

where $R_{B}=10^{-6} \mathrm{~m}$ is the generalized bubble radius, $p_{v}=$ $2340 \mathrm{~Pa}$ is the saturated vapor pressure, $a_{\text {nuc }}=5 \times 10^{-4}$ is the nucleation site volume fraction, $F_{\text {vap }}=50$ is the evaporation coefficient, and $F_{\text {cond }}=0.01$ is the condensation coefficient.

3.2 Large Eddy Simulation Approach. LES equations are derived from the above Eqs. (2) and (3) by applying a Favrefiltering operation

$$
\frac{\partial \rho}{\partial t}+\frac{\partial\left(\rho \bar{u}_{j}\right)}{\partial x_{j}}=0
$$

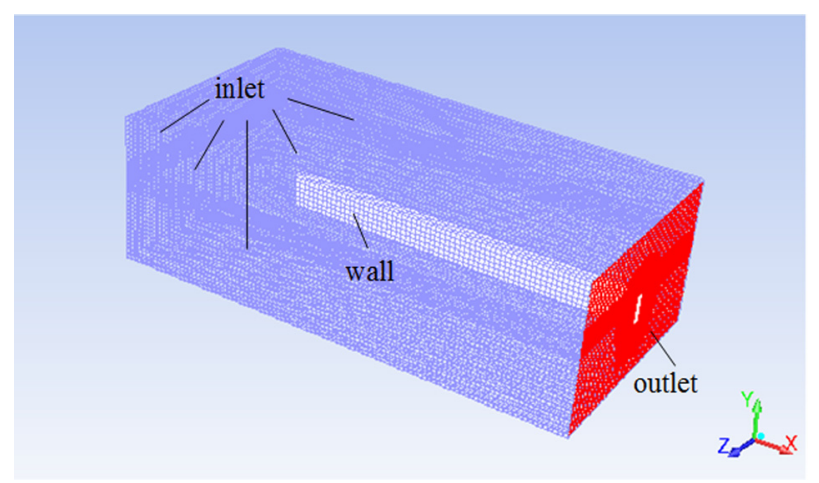

Fig. 8 Calculated domain and boundary conditions. Boundary conditions that contain inlet, outlet, and wall are marked.

$$
\frac{\partial\left(\rho \bar{u}_{i}\right)}{\partial t}+\frac{\partial\left(\rho \bar{u}_{i} \bar{u}_{j}\right)}{\partial x_{j}}=-\frac{\partial \bar{p}}{\partial x_{j}}+\frac{\partial}{\partial x_{j}}\left(\mu \frac{\partial \bar{u}_{j}}{\partial x_{j}}\right)-\frac{\partial \tau_{i j}}{\partial x_{j}}
$$

where $\tau_{i j}$ is the subgrid scale (SGS) stress, which is defined as

$$
\tau_{i j}=\rho\left(\overline{u_{i} u_{j}}-\bar{u}_{i} \bar{u}_{j}\right)
$$

Based on the Boussinesq equation, the SGS stress could be computed from

$$
\tau_{i j}-\frac{1}{3} \tau_{k k} \delta_{i j}=-2 \mu_{t} \bar{S}_{i j}
$$

where $\mu_{t}$ is the eddy viscosity, $\tau_{k k}$ is the isotropic part, and $\bar{S}_{i j}$ is the rate-of-strain which is defined as

$$
\bar{S}_{i j} \equiv \frac{1}{2}\left(\frac{\partial \bar{u}_{i}}{\partial x_{j}}+\frac{\partial \bar{u}_{j}}{\partial x_{i}}\right)
$$

In the wall adapting local eddy viscosity (WALE) model, the eddy viscosity is modeled by

$$
\mu_{t}=\rho \Delta_{s}^{2} \frac{\left(S_{i j}^{d} S_{i j}^{d}\right)^{3 / 2}}{\left(\bar{S}_{i j} \bar{S}_{i j}\right)^{5 / 2}+\left(S_{i j}^{d} S_{i j}^{d}\right)^{5 / 4}}
$$

where $\Delta_{s}=C_{w} V^{1 / 3}, \quad S_{i j}^{d}=(1 / 2)\left(\bar{g}_{i j}^{2}+\bar{g}_{i j}^{2}\right)-(1 / 3) \delta_{i j} \bar{g}_{k k}^{2}, \quad \bar{g}_{i j}$ $=\left(\partial \bar{u}_{i} / \partial x_{j}\right)$, and the constant $C_{w}=0.325$.

3.3 Simulation Setups. The Cartesian cut-cell method, which has witnessed significant development in recent years, presents an effective approach for generating an unstructured mesh. This approach easily achieves local refinement and perfect orthogonality and is suitable for complex geometries $[39,40]$.

A semi-infinite model is adopted to overcome the effect of the hydrofoil tail. The commercial software, ANSYS meshing, is used to generate a Cartesian cut-cell mesh with 15 layers of inflation. The height of the first layer is set to $2 \times 10^{-4} \mathrm{~m}$ with a 1.1 growth rate, and the model has a minimum face size of $2 \times 10^{-3} \mathrm{~m}$. The total cell number is about 3 million.

The commercial software, FLUENT, is used for the simulation. Figure 8 presents the defined boundary conditions, including the velocity inlet, pressure outlet, and wall.

Large eddy simulation and the WALE model are adopted to simulate the turbulent flow. Table 1 shows the other detailed numerical schemes and parameters. Second-order implicit scheme is used for time discretization of the volume fraction equation, which is compatible with the cavitation model. The body force weighted option is selected for pressure interpolation. The modified high-resolution interface-capturing scheme selected is more robust than the explicit geometric reconstruction scheme. As the whole acceleration process is very short in the experiment, the 
Table 1 Numerical schemes and parameters

\section{Scheme in time}

Pressure interpolation

Scheme in momentum

Scheme in volume fraction

Time step size
Second-order implicit

Pressure staggering option (PRESTO!)

Bounded central differencing

Modified high resolution interface capturing (HRIC) $1 \times 10^{-5}$

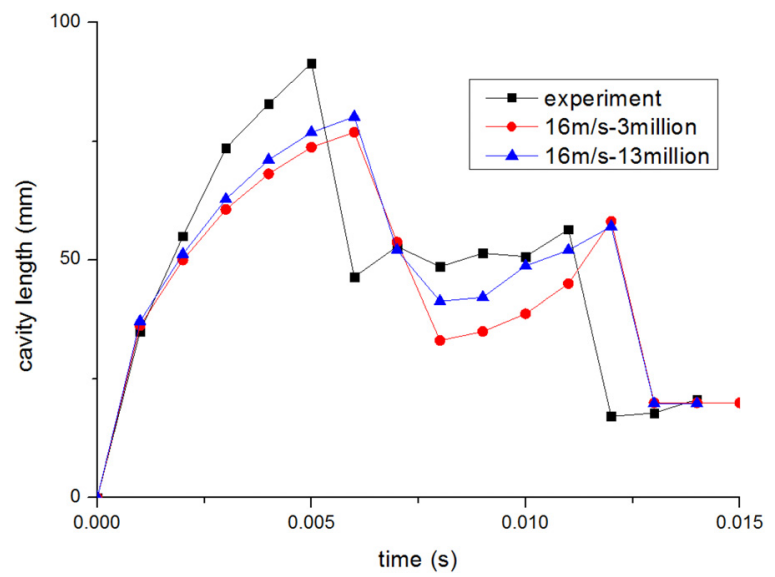

Fig. 9 Comparison of the cavity length among the experimental, original mesh, and refined mesh simulated results

unsteady cavitating flow simulations are started from a uniform flow field. The time step size is set as $10^{-5} \mathrm{~s}$.

3.4 Mesh Independence Study. To verify the suitability of the original mesh size for the simulation, we produce a new refined mesh that contains about 13 million cells. We employ the same Cartesian cut-cell finite-volume approach.

The resultant cavity lengths in the previous simulation are compared with those in the experiment. Figure 9 presents the comparison results. Although the refined mesh contains more detailed flow structures, we consider the main features of cavity evolution to a greater extent than the other attributes. By comparing the images in Fig. 10, we find that the refined mesh simulation results are consistent with the original results for cavity evolution. The features used for the comparison include cavity length, re-entry jet fronts, and cavity shape. After verifying the mesh independence of the simulation method, the simulation results of the original mesh are used for further analysis and discussion. The numerical method with a Cartesian cut-cell mesh is stable, and the small cell sizes have fine simulated results.

\section{Results and Discussion}

4.1 Simulation of Various Constant Speed Cases. As mentioned in Sec. 2.1, the speed of the model varies from about $26 \mathrm{~m} / \mathrm{s}$ to $16 \mathrm{~m} / \mathrm{s}$. Therefore, we present the results of those simulations with various constant speeds to find how the cavity length and evolution process change along with speed. Figure $11 \mathrm{com}-$ pares the experiment and simulation results of cases with constant speeds of $20 \mathrm{~m} / \mathrm{s}, 18 \mathrm{~m} / \mathrm{s}$, and $16 \mathrm{~m} / \mathrm{s}$, and some differences are observed among these cases. Cavity length increases along with the model speed, but the whole cavity evolution process (i.e., the period) does not change significantly. Although the period of the simulation results does not exactly match that of the experimental results, both sets of results are similar in terms of general regularity. A re-entry jet is generated at around $t=0.007 \mathrm{~s}$ in the simulated cases, thereby leading to the partial shedding of the cavity. Given that the cavity length increases at a higher speed, the reentry jet takes a longer time to reach the leading edge of the hydrofoil.

Figure 12 shows the cavitating flow around the hydrofoil evolution during the simulation and experiment from $t=0.002 \mathrm{~s}$ to $0.014 \mathrm{~s}$. Given the huge deceleration of the hydrofoil in the water tank experiment, the front and latter part speeds of the simulation are smaller and larger than those of the experiment, respectively. The arrows indicate that the main features contain the partial shed cavity, the horseshoe cavity structure induced by the re-entry jet, and the re-entry jet that is deflected from the free surface and is located parallel to the free surface. Moreover, the partial cavity

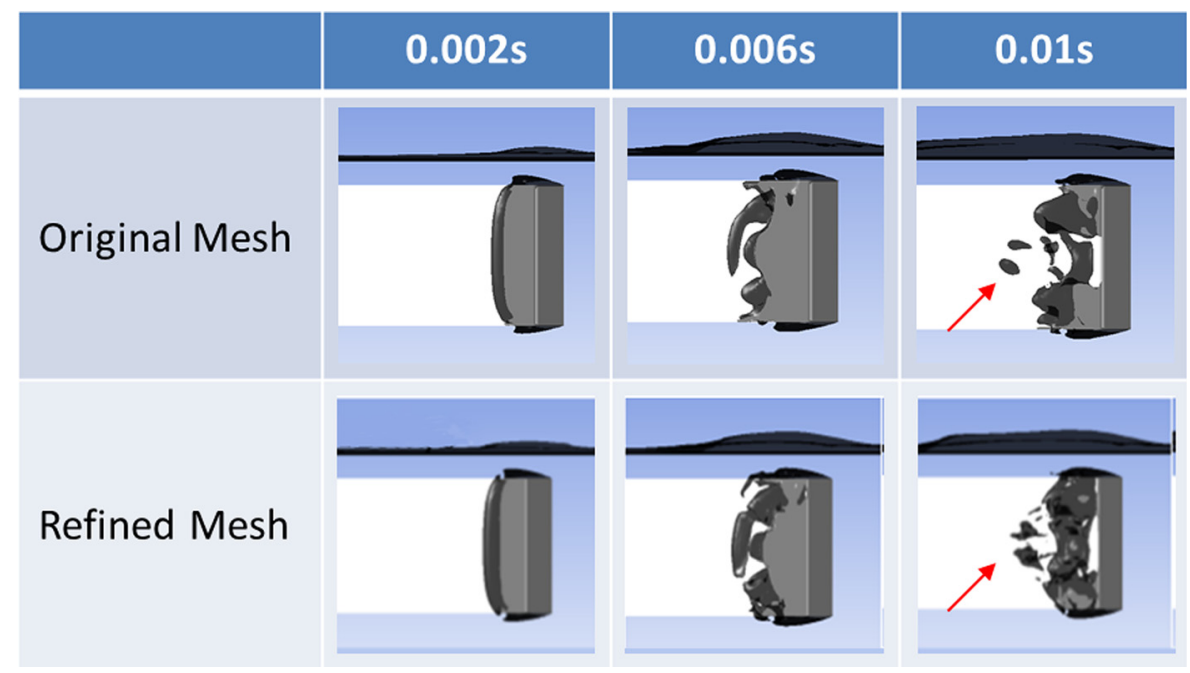

Fig. 10 Comparison of the cavity evolution between the original mesh and refined mesh simulated results. The detailed structure of the cavity is shown and pointed out by the arrows. 


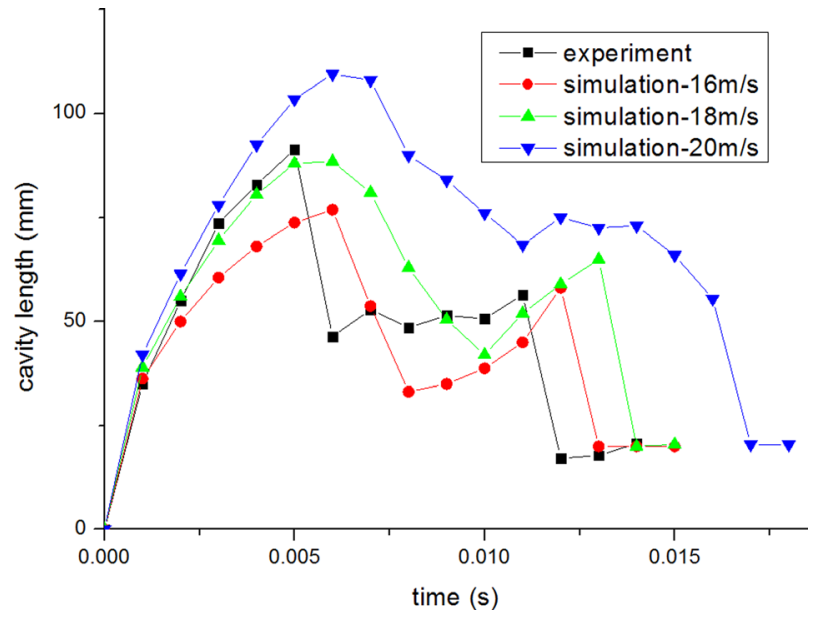

Fig. 11 Comparison of the cavity length between the experiment results and the simulation results of cases with constant speeds of $16 \mathrm{~m} / \mathrm{s}, 18 \mathrm{~m} / \mathrm{s}$, and $20 \mathrm{~m} / \mathrm{s}$

shedding (i.e., the moment) and re-entry jet appear in the middle of the model at almost the same time in all three simulated cases.

The re-entry jet later reaches the leading edge of the hydrofoil as the simulated speed and cavity length increase. The velocity of the re-entry jet may also be affected by the changing speed. Figure 13 compares the simulation results of cases with constant speeds of 16,18 , and $20 \mathrm{~m} / \mathrm{s}$ in terms of the length of the re-entry jet from the end of the main cavity to the front of the re-entry jet inside the cavity. The velocity of the re-entry jet (i.e., slope of the lines) is higher at the beginning and remains almost the same for the three cases. However, the velocity becomes slightly lower at the end for the cases with constant speeds of $18 \mathrm{~m} / \mathrm{s}$ and $20 \mathrm{~m} / \mathrm{s}$. Therefore, the re-entry jet takes a long time to reach the leading edge of the hydrofoil because of the increasing cavity length and the decreasing speed of the re-entry jet.

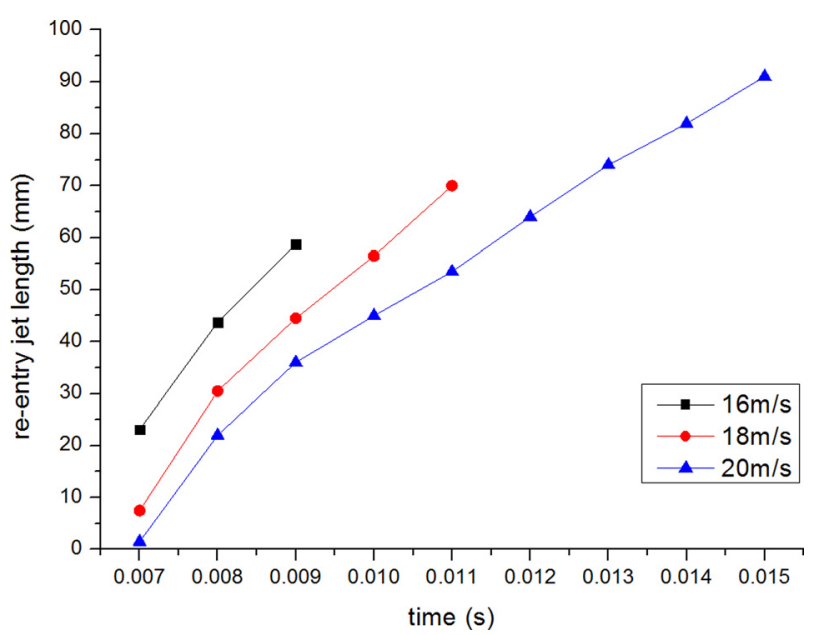

Fig. 13 Comparison of the re-entry jet length among the simulation results of $16 \mathrm{~m} / \mathrm{s}, 18 \mathrm{~m} / \mathrm{s}$, and $20 \mathrm{~m} / \mathrm{s}$

4.2 Free Surface Effect. To analyze the free surface effect, this paper simulates and compares two cases with and without a free surface at a $16 \mathrm{~m} / \mathrm{s}$ model speed. The main features of the cavity during the evolution process are also analyzed. First, the structure of the broken ring at the end of the cavity at $t=0.004 \mathrm{~s}$ as shown in the water tank experiment image is compared with the simulation results with and without a free surface at $t=0.006 \mathrm{~s}$. Figure 14 presents the comparison results. The pressure contour chart on the hydrofoil surface is also presented to show the structure clearly. Similar to the experimental results, the broken ring moves near the upper side of the hydrofoil in the simulated case with a free surface. The middle part of the cavity is longer than the rest because of the edge effects on both sides, which can be bypassed. Therefore, the upper and lower sides of the hydrofoil have a small negative pressure, while its middle part has a strong constraint and a high negative pressure. Therefore, the cavity length is increased. The

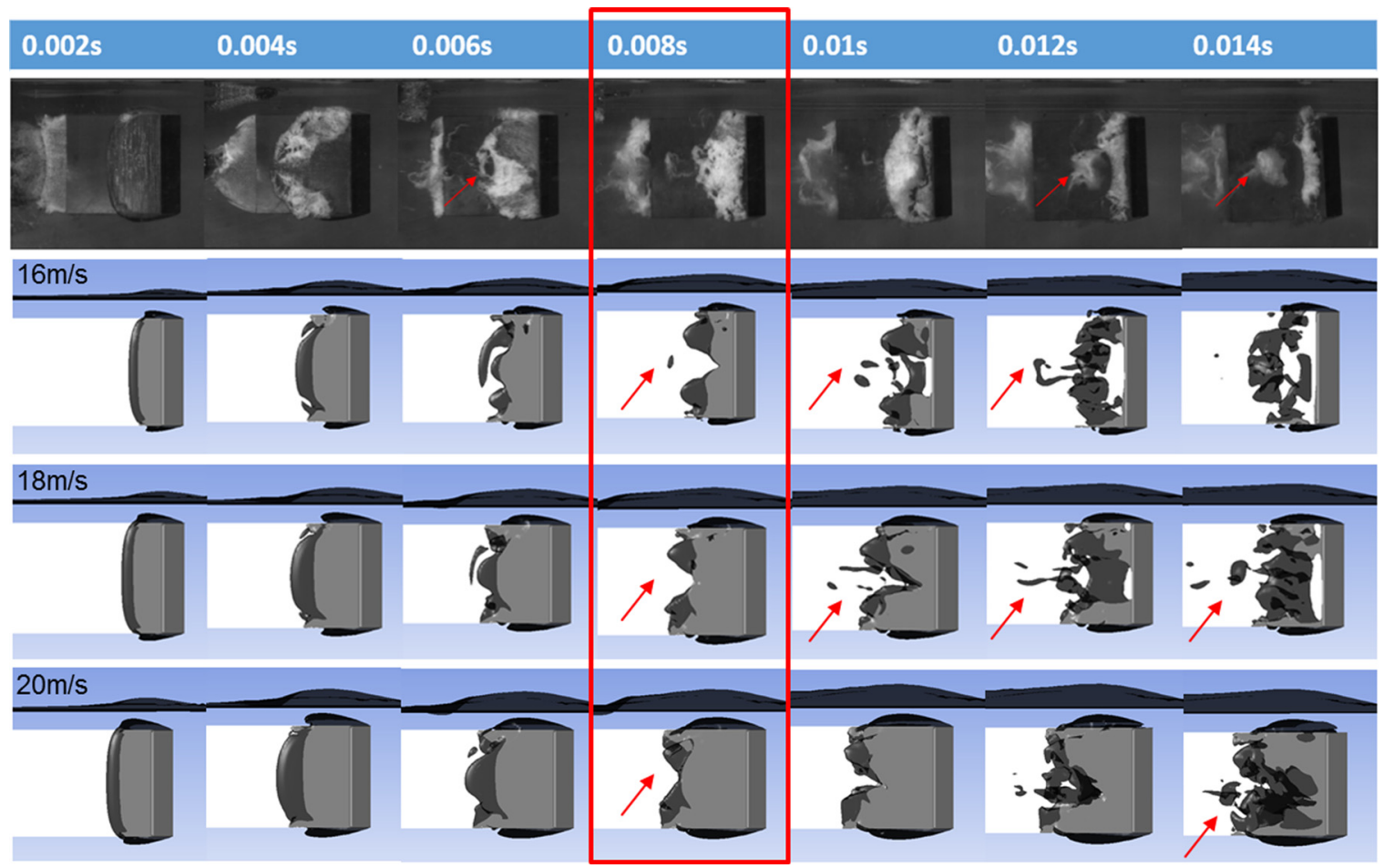

Fig. 12 Resulted cavity evolution process of the simulated cases and water tank experiment. The characteristics of the cavitating flow are pointed out by the arrows. 

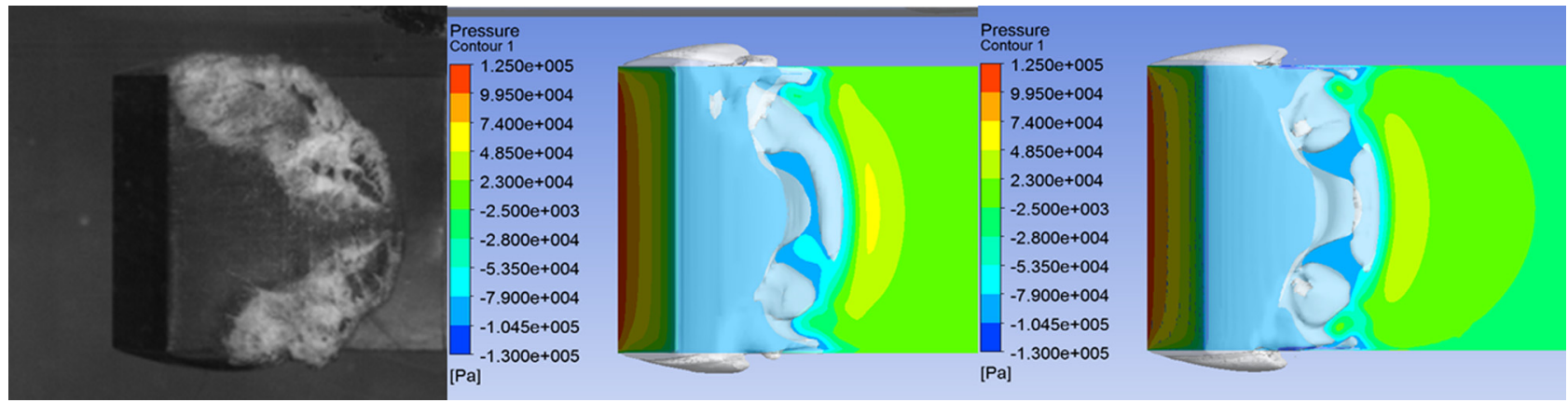

Fig. 14 Comparison of the structure of the broken bubble ring at the end of the cavity at $t=0.004 \mathrm{~s}$ in the water tank experiment and at $t=0.006 \mathrm{~s}$ for the simulation results with and without free surface when the model speed is $16 \mathrm{~m} / \mathrm{s}$. Pressure distribution is shown on the surface of the hydrofoil.

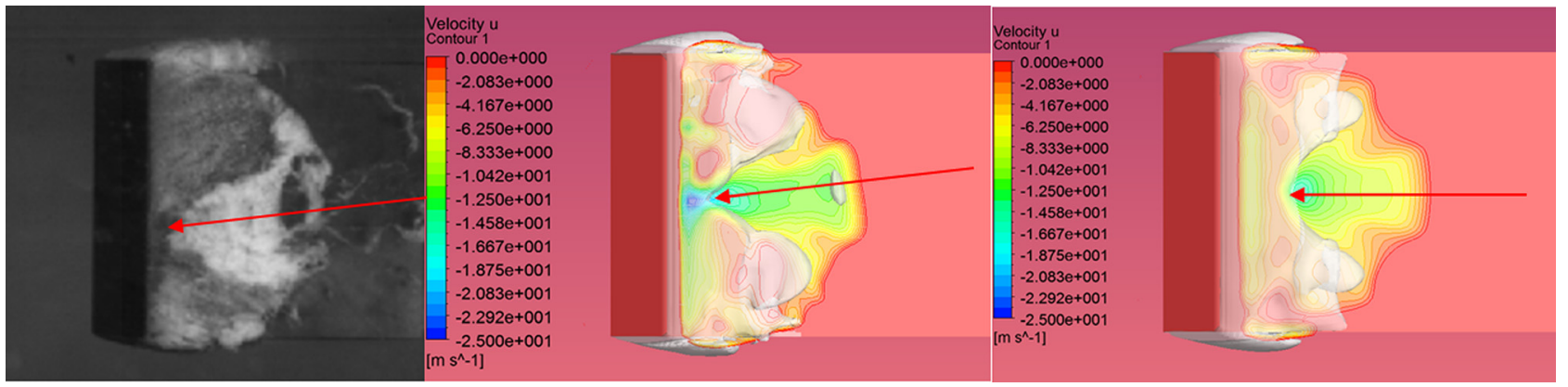

Fig. 15 Comparison of the experiment image at $t=0.006 \mathrm{~s}$ and the velocity contour chart of the cases with and without free surface for the model speed at $16 \mathrm{~m} / \mathrm{s}$ at $t=0.008 \mathrm{~s}$. The direction of the re-entry jet inside the cavity is pointed out by the arrows.
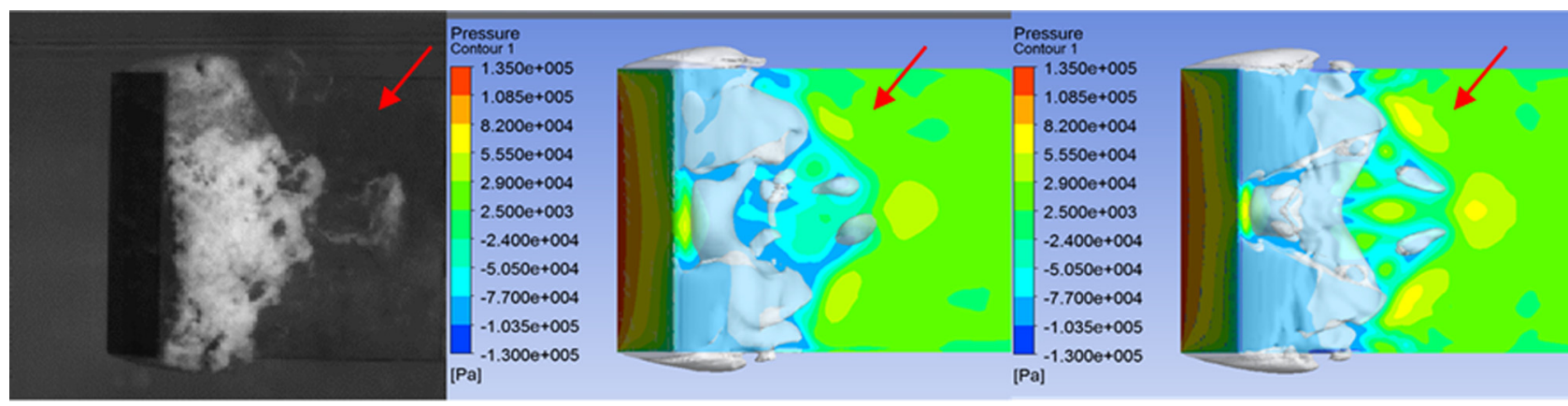

Fig. 16 Comparison of the horseshoe cavity structure at $t=0.008 \mathrm{~s}$ in the water tank experiment and at $t=0.01 \mathrm{~s}$ for the simulation results with and without free surface when the model speed is $16 \mathrm{~m} / \mathrm{s}$. Pressure distribution on the surface of the hydrofoil is shown. The horseshoe cavity structure induced by the re-entry jet is pointed out by the arrows.

case with a free surface has a weak upward constraint and a strong edge effect under the free surface effect, thereby moving the cavity downward.

As mentioned above, the re-entry jet inside the cavity does not move toward the center of the cavity sheet, but is deflected from the free surface under the free surface effect. To see the position of the re-entry jet clearly, Fig. 15 presents the contour chart for the velocity around the model in the $x$ direction at $t=0.008 \mathrm{~s}$ by adding a plane at $y=0.021 \mathrm{~m}$ and comparing the results of the cases with and without a free surface. The arrows indicate the direction of the re-entry jet. The experimental image at $t=0.006 \mathrm{~s}$ is also presented. A black straight line is added along the center of the model. The figures exclude the value of velocity. The case with a free surface has a strong upward reverse pressure gradient, thereby moving the whole re-entry jet downward.

Figure 16 presents the horseshoe cavity structure induced by the re-entry jet. The water tank experiment image at $t=0.008 \mathrm{~s}$ and the simulation results with and without a free surface at $t=0.01 \mathrm{~s}$ are presented for comparison. The pressure contour chart on the hydrofoil surface is also presented. The horseshoe cavity structure appears in both simulated cases. The cavity structure of the case without a free surface is axisymmetric during the whole period.

4.3 Effect on Shedding Vortex Structures. The relationship between the shedding cavity and vortex motion can be visualized based on the $Q$-criterion, which is defined as follows [41]:

$$
Q=0.25 \times(W \times W-S \times S)
$$

where $W=\left(2 W_{i j} \times W_{i j}\right)^{0.5}$ is the vorticity magnitude (scalar), $S=\left(2 S_{i j} \times S_{i j}\right)^{0.5}$ is the rate-of-strain (scalar), $S_{i j}$ is the mean rate of the strain tensor, and $W_{i j}$ is the mean vorticity tensor. $S_{i j}$ and $W_{i j}$ are the symmetric and antisymmetric part of the mean velocity 


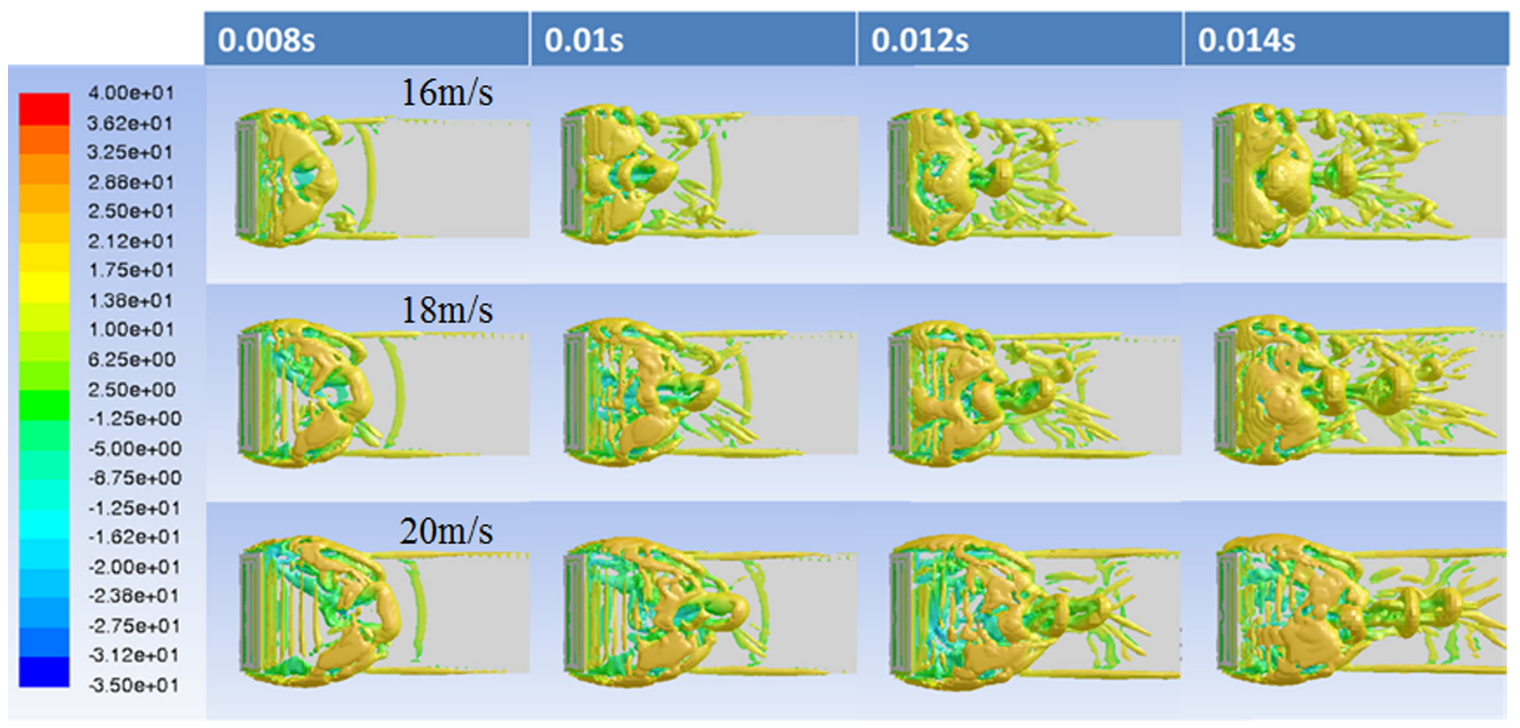

Fig. 17 Velocity distribution on the added isosurface of $Q=50,000 \mathrm{~s}^{-2}$ at $t=0.008 \mathrm{~s}, t=0.01 \mathrm{~s}, t=0.012 \mathrm{~s}$, and $t=0.014 \mathrm{~s}$ for the simulated cases at constant speed $16 \mathrm{~m} / \mathrm{s}, 18 \mathrm{~m} / \mathrm{s}$, and $20 \mathrm{~m} / \mathrm{s}$

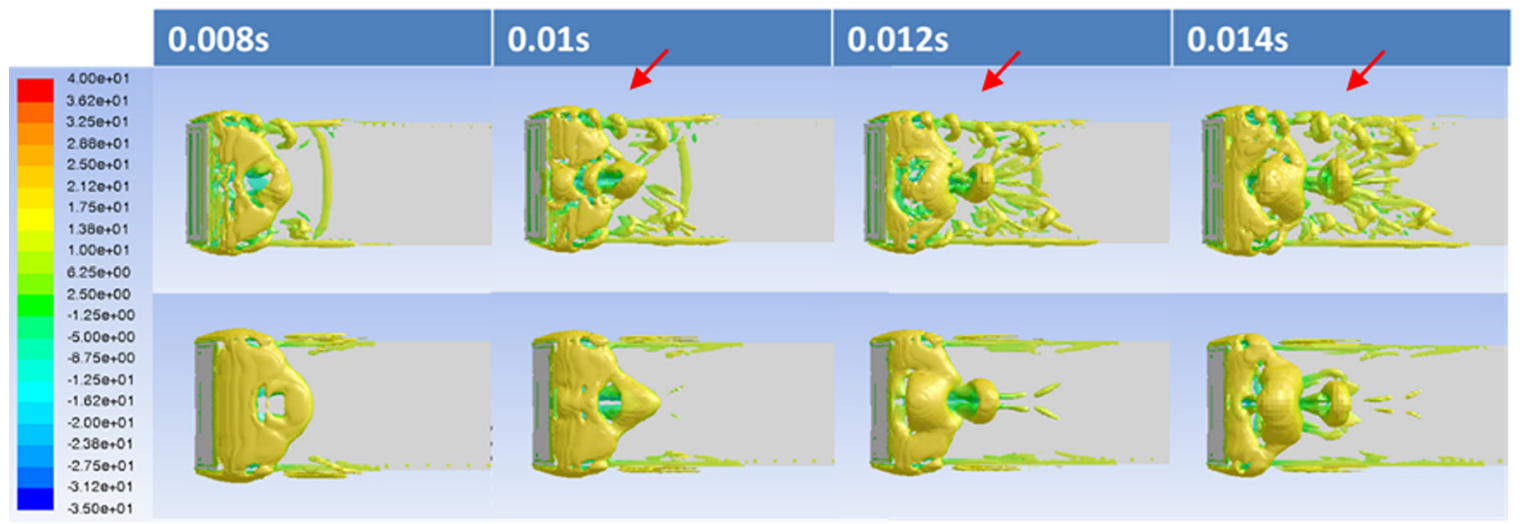

Fig. 18 Velocity distribution on the added isosurface of $Q=50,000 \mathrm{~s}^{-2}$ at $t=0.008 \mathrm{~s}, t=0.01 \mathrm{~s}, t=0.012 \mathrm{~s}$, and $t=0.014 \mathrm{~s}$ of the simulated cases with and without free surface when the model speed is $16 \mathrm{~m} / \mathrm{s}$

gradient tensor, respectively. The second invariant of the velocity gradient tensor is defined as $Q=0.5 \times\left(W_{i j} \times W_{i j}-S_{i j} \times S_{i j}\right)$.

Figure 17 shows the velocity distribution on the added isosurface of $Q=50,000 \mathrm{~s}^{-2}$ at $t=0.008,0.01,0.012$, and $0.014 \mathrm{~s}$ for the simulated cases at constant speeds of 20,18 , and $16 \mathrm{~m} / \mathrm{s}$. The figure also shows the cavity shedding and collapsing stages of the cavity evolution process. Apart from the nonaxisymmetric part of the broken vortex around the hydrofoil, Fig. 17 also shows that the broken vortex becomes larger as the simulation speed increases.

Figure 18 presents the velocity distribution on the added isosurface of $Q=50,000 \mathrm{~s}^{-2}$ for those simulated cases with and without a free surface at a model speed of $16 \mathrm{~m} / \mathrm{s}$. For the case without a free surface, the broken vortex around the hydrofoil is axisymmetric. When the model is positioned near the free surface, the broken vortex becomes thicker on the upper side of the model under the effect of the free surface. The nonaxisymmetric vortex around the projectile caused by the collapse of the shedding cavity can generate a large lateral force and influence the cruising stability. Apart from the vortex position, the smoothness of the vortices becomes worse when the free surface is located nearby, thereby suggesting that some small-scale disturbances may be introduced.

The three-dimensional vorticity transport equation is used to analyze the interaction between the vortex and cavitation. The simulated cases with and without a free surface at a $16 \mathrm{~m} / \mathrm{s}$ model speed are also presented to understand the effect of the free surface on the vortex [42-44]

$$
\frac{D \boldsymbol{\omega}}{D t}=(\boldsymbol{\omega} \cdot \nabla) \boldsymbol{V}-\boldsymbol{\omega}(\nabla \cdot \boldsymbol{V})+\frac{\nabla \rho_{m} \times \nabla p}{\rho^{2}{ }_{m}}+\frac{1}{\operatorname{Re}}\left(\nabla^{2} \boldsymbol{\omega}\right)
$$

where $D / D t$ is the material derivative operator, $\omega$ is flow vorticity, $V$ is the flow velocity, $\rho$ is the local fluid density, $p$ is the local pressure, Re is Reynolds number and $\nabla^{2}$ is the Laplace operator.

The four terms in the equation, namely, vortex stretching, volumetric expansion/contraction (dilatation), baroclinic torque (resulting from the misaligned pressure and density gradients), and viscous diffusion, represent the physical mechanism of the vorticity variation of fluid particles during their motion. Given its much smaller effect on vorticity transport than the other terms for the high Reynolds number flow, the last term in the equation can be ignored during the analysis.

Figures 19-21 present the velocity distribution on the added isosurface when the calculated vortex-stretching, vortexdilatation, and baroclinic torque magnitudes of the simulated cases are equal to 50,000 at various times when the model speed is set to $16 \mathrm{~m} / \mathrm{s}$. The cases with and without a free surface show obvious differences. Figure 19 shows that the case with a free surface has a highly complex vortex-stretching magnitude structure. Disturbances occur on the upper side of the hydrofoil near the free 


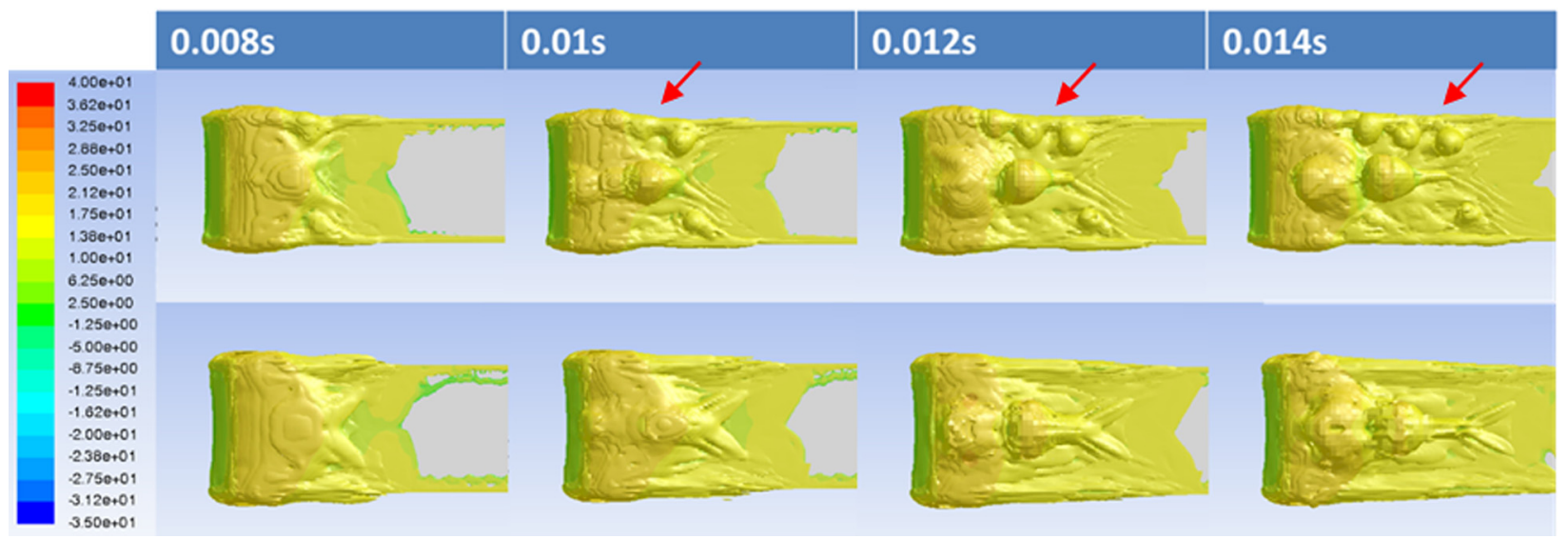

Fig. 19 Velocity distribution on the added isosurface of vortex-stretching magnitude is 50,000 at $t=0.008 \mathrm{~s}, t=0.01 \mathrm{~s}$, $t=0.012 \mathrm{~s}$, and $t=0.014 \mathrm{~s}$ of the simulated cases with and without free surface when the model speed is $16 \mathrm{~m} / \mathrm{s}$

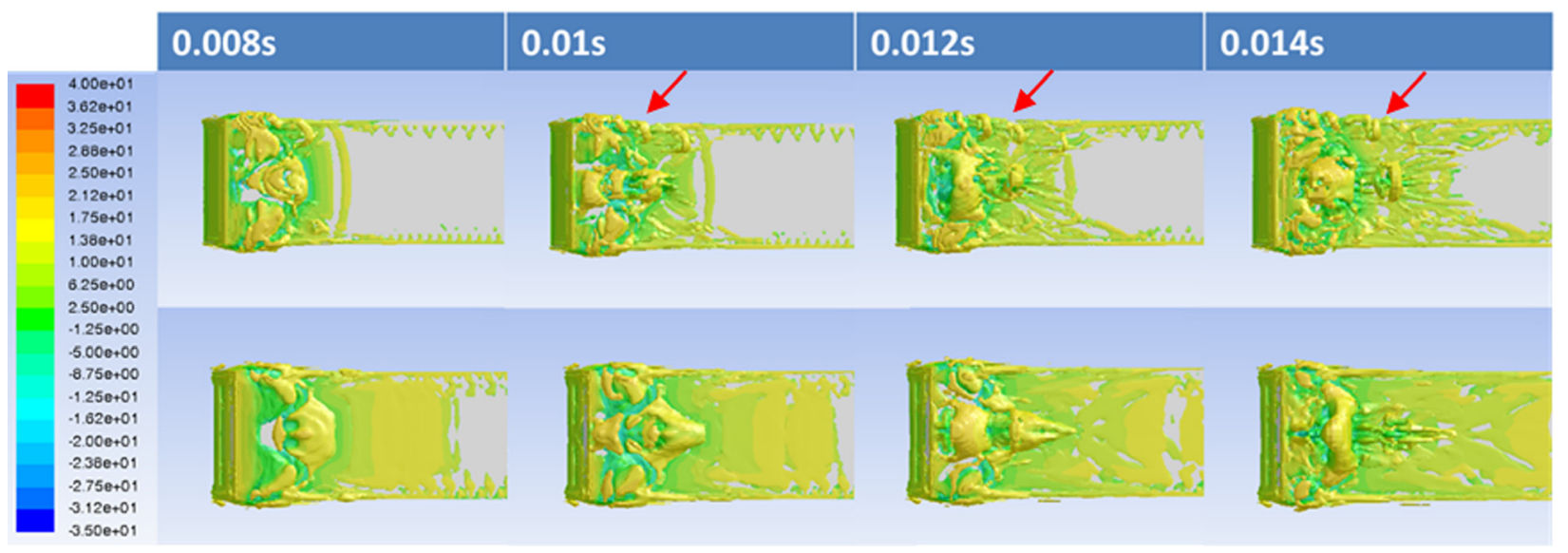

Fig. 20 Velocity distribution on the added isosurface of vortex-dilatation magnitude is 50,000 at $t=0.008 \mathrm{~s}, t=0.01 \mathrm{~s}$, $t=0.012 \mathrm{~s}$, and $t=0.014 \mathrm{~s}$ of the simulated cases with and without free surface when the model speed is $16 \mathrm{~m} / \mathrm{s}$

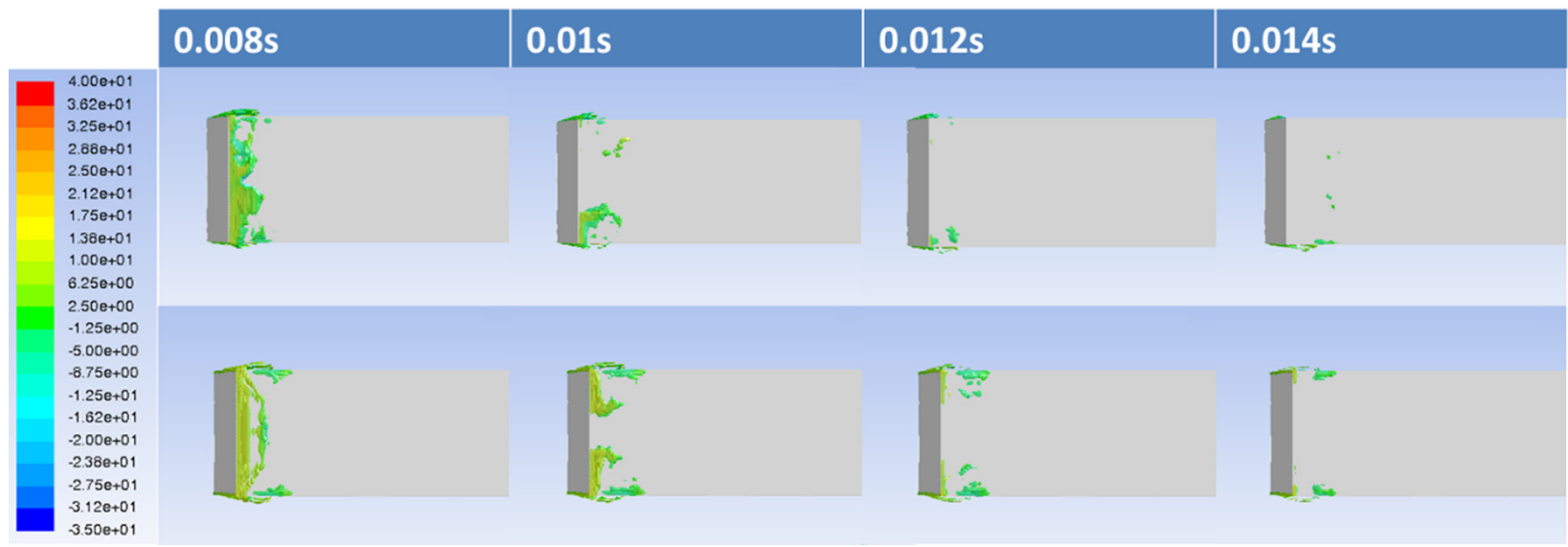

Fig. 21 Velocity distribution on the added isosurface of baroclinic torque magnitude is 50,000 at $t=0.008 \mathrm{~s}, t=0.01 \mathrm{~s}$, $t=0.012 \mathrm{~s}$, and $t=0.014 \mathrm{~s}$ of the simulated cases with and without free surface when the model speed is $16 \mathrm{~m} / \mathrm{s}$

surface. The vortex-dilatation magnitude of the simulated cases in Fig. 20 also shows many small-scale disturbances in the case with a free surface. The structure of the case with a free surface is also more broken than that of the case without a free surface. Figure 21 shows that the baroclinic torque magnitude has a small drawn isosurface that does not dominate the results of the vortex around the hydrofoil.

Therefore, the main factor lies in the first term, namely, the vortex-stretching magnitude. Given the small upper constraint under the effect of free surface, the velocity gradient becomes larger and the shedding vortex tends to be stretched toward the final horseshoe profile. The second term, vortex-dilatation magnitude, reflects the density change in the continuity equation, which involves the formation and phase transition of the cavity. Therefore, the presented characteristics in Fig. 20 are the same as those of the original form of the cavity. Moreover, when the cavity is generated, the vortex-dilatation magnitude produces a negative effect, thereby weakening the original vortex. The condensed 
vortex gradually becomes stronger as the generated vortex becomes weaker. The third term, baroclinic torque, reflects the inconsistencies in the direction of pressure and density gradient. This term has a small value because the density change is driven by the pressure change in the flow-dominated phase transition.

\section{Conclusions}

A new physical problem about the cloud cavitating flow that surrounds an underwater-launched hydrofoil near the free surface at relatively high Froude number is discussed in this paper. Water tank experiment and CFD simulation are performed using LES with Cartesian cut-cell mesh method to analyze the problem. The experimental and simulated results agree well with each other.

Asymmetric cavity around the hydrofoil can be observed through the results, which show the evolution of the cavitating flow under free surface effect. The free surface nearby induce relatively weak upward constraint, thereby moving the whole cavity downward. Otherwise, under the free surface effect, the re-entry jet inside the cavity does not move toward the center of the cavity sheet, but is deflected away from the free surface. Simulation shows that the hydrofoil with a free surface nearby experiences a strong upward reverse pressure gradient, thereby moving the whole re-entry jet downward.

The regularity between the cavity and vortex motion can be visualized using the $Q$-criterion. The nonaxisymmetric vortex around the hydrofoil is generated by the collapse of the shedding cavity under the free surface effect, which may affect cruising stability. The broken vortex becomes larger as the simulation speed increases. Terms in the vorticity transport equation are separately analyzed to identify the dominant physical mechanism. Major differences are observed in the results for vortex stretching and dilatation, thereby suggesting that some small-scale disturbances are introduced near the free surface.

Free surface has complex effects on the cavity around a hydrofoil, and such effects may change along with the distance between the upper side of the hydrofoil and the free surface. The numerical methods used in this article are relatively stable and can be used to solve other similar near-surface high-speed cavitating flow problems. In future engineering work, people may need to pay attention to the asymmetric effect of the cavitating flow brought by the free surface. The results in this study are limited to typical working conditions for a typically shaped model, thereby requiring further in-depth analysis.

\section{Funding Data}

- The authors would like to thank the National Natural Science Foundation of China through Grant Nos. 11202215, 11332011, and 11672315 .

- This project was also supported by the Youth Innovation Promotion Association CAS (Grant No. 2015015).

\section{Nomenclature}

$$
\begin{aligned}
a_{\text {nuc }} & =\text { nucleation site volume fraction } \\
d & =\text { diameter of the projectile } \\
F_{\text {cond }} & =\text { condensation coefficient } \\
F_{\text {vap }} & =\text { evaporation coefficient } \\
g & =\text { gravity } \\
l & =\text { liquid water } \\
\dot{m}^{+} & =\text {mass transfer rate of evaporation } \\
\dot{m}^{-} & =\text {mass transfer rate of condensation } \\
p & =\text { pressure } \\
p_{v} & =\text { saturated vapor pressure } \\
p_{\infty} & =\text { standard atmospheric pressure } \\
R_{B} & =\text { generalized bubble radius } \\
S_{i j} & =\text { mean rate of the strain tensor } \\
u_{i} & =\text { velocity component in } i \text { direction } \\
v & =\text { water vapor } \\
W_{i j} & =\text { mean vorticity tensor }
\end{aligned}
$$

$\alpha=$ volume fraction of the different phases

$\delta_{i j}=$ Kronecker symbol

$\mu=$ laminar viscosity

$\mu_{t}=$ eddy viscosity

$v_{\infty}=$ launch speed

$\rho=$ mixture density

$\rho_{l}=$ liquid water density

$\sigma=$ cavitation number

$\tau_{i j}=$ SGS stress

$\tau_{k k}=$ isotropic part

\section{References}

[1] Knapp, R. T., Daily, J. W., and Hammitt, F. G., 1970, Cavitation, McGrawHill, New York.

[2] Brennen, C. E., 2013, Cavitation and Bubble Dynamics, Cambridge University Press, Cambridge, UK.

[3] Peng, X., Ji, B., Cao, Y., Xu, L., Zhang, G., Luo, X., and Long, X., 2016, "Combined Experimental Observation and Numerical Simulation of the Cloud Cavitation With U-Type Flow Structures on Hydrofoils," Int. J. Multiphase Flow, 79, pp. 10-22.

[4] Wei, Y., Wang, Y., Fang, X., Huang, C., and Duan, Z., 2011, "A Scaled Underwater Launch System Accomplished by Stress Wave Propagation Technique," Chin. Phys. Lett., 28(2), p. 024601.

[5] Kanfoudi, H., Lamloumi, H., and Zgolli, R., 2012, "Numerical Investigation for Steady and Unsteady Cavitating Flows," Advances in Modeling of Fluid Dynamics, InTech, Rijeka, Croatia.

[6] Wu, Q., Huang, B., and Wang, G., 2015, "Numerical Simulation of Transient Flows Around a 3D Pitching Hydrofoil," Adv. Mech. Eng., 7(2), p. 808034.

[7] Wang, Y., Huang, C., Fang, X., Yu, X., Wu, X., and Du, T., 2016, "Cloud Cavitating Flow Over a Submerged Axisymmetric Projectile and Comparison Between Two-Dimensional RANS and Three-Dimensional Large-Eddy Simulation Methods," ASME J. Fluids Eng., 138(6), p. 061102.

[8] Yu, X., Huang, C., Du, T., Liao, L., Wu, X., Zheng, Z., and Wang, Y., 2014 "Study of Characteristics of Cloud Cavity Around Axisymmetric Projectile by Large Eddy Simulation,” ASME J. Fluids Eng., 136(5), p. 051303.

[9] Bensow, R. E., and Bark, G., 2010, "Implicit LES Predictions of the Cavitating Flow on a Propeller," ASME J. Fluids Eng., 132(4), p. 041302.

[10] Pendar, M.-R., and Roohi, E., 2016, "Investigation of Cavitation Around 3D Hemispherical Head-Form Body and Conical Cavitators Using Different Turbulence and Cavitation Models," Ocean Eng., 112, pp. 287-306.

[11] Hidalgo, V., Luo, X., Escaler, X., Ji, J., and Aguinaga, A., 2014, "Numerical Investigation of Unsteady Cavitation Around a NACA 66 Hydrofoil Using OpenFOAM," J. Phys.: Conf. Ser., 22, p. 052013.

[12] Gnanaskandan, A., and Mahesh, K., 2015, "Large Eddy Simulation of Turbulent Cavitating Flows," J. Phys. Conf. Ser., 656(1), p. 012135.

[13] Egerer, C. P., Schmidt, S. J., Hickel, S., and Adams, N. A., 2016, "Efficient Implicit LES Method for the Simulation of Turbulent Cavitating Flows," J. Comput. Phys., 316, pp. 453-469.

[14] Egerer, C. P., Hickel, S., Schmidt, S. J., and Adams, N. A., 2014, "Large-Eddy Simulation of Turbulent Cavitating Flow in a Micro Channel," Phys. Fluids, 26(8), p. 085102.

[15] Ma, J., Hsiao, C.-T., and Chahine, G. L., 2015, "Shared-Memory Parallelization for Two-Way Coupled Euler-Lagrange Modeling of Cavitating Bubbly Flows," ASME J. Fluids Eng., 137(12), p. 121106.

[16] Gnanaskandan, A., and Mahesh, K., 2016, "Numerical Investigation of NearWake Characteristics of Cavitating Flow Over a Circular Cylinder," J. Fluid Mech., 790, pp. 453-491.

[17] Kim, J., and Lee, J. S., 2015, "Numerical Study of Cloud Cavitation Effects on Hydrophobic Hydrofoils,” Int. J. Heat Mass Transfer, 83, pp. 591-603.

[18] Hidalgo, V., Luo, X.-W., Escaler, X., Bin, J., and Aguinaga, A., 2015, "Implicit Large Eddy Simulation of Unsteady Cloud Cavitation Around a Plane-Convex Hydrofoil," J. Hydrodyn, Ser. B, 27(6), pp. 815-823.

[19] Chen, Y., Chen, X., Gong, Z., Li, J., and Lu, C., 2016, "Numerical Investigation on the Dynamic Behavior of Sheet/Cloud Cavitation Regimes Around Hydrofoil," Appl. Math. Model., 40(11), pp. 5835-5857.

[20] Ji, B., Luo, X., Peng, X., Wu, Y., and Xu, H., 2012, "Numerical Analysis of Cavitation Evolution and Excited Pressure Fluctuation Around a Propeller in Non-Uniform Wake," Int. J. Multiphase Flow, 43, pp. 13-21.

[21] Li, L., Jia, Q., Liu, Z., Li, B., Hu, Z., and Lin, Y., 2015, "Eulerian Two-Phase Modeling of Cavitation for High-Speed UUV Using Different Turbulence Models," IEEE International Conference on Cyber Technology in Automation, Control, and Intelligent Systems (CYBER), Shenyang, China, June 8-12, pp. 1247-1252.

[22] Ji, B., Peng, X., Long, X., Luo, X., and Wu, Y., 2015, "Numerical Evaluation of Cavitation Shedding Structure Around 3D Hydrofoil: Comparison of PANS, LES and RANS Results With Experiments," J. Phys. Conf. Ser., 656(1), p. 012127.

[23] Akcabay, D. T., and Young, Y. L., 2014, "Influence of Cavitation on the Hydroelastic Stability of Hydrofoils," J. Fluids Struct., 49, pp. 170-185.

[24] Faltinsen, O. M., 2005, Hydrodynamics of High Speed Marine Vehicles, Cambridge University Press, Cambridge, UK.

[25] Faltinsen, O. M., and Semenov, Y. A., 2008, "The Effect of Gravity and Cavitation on a Hydrofoil Near the Free Surface,” J. Fluid Mech., 597, pp. 371-394. 
[26] Bal, S., and Kinnas, S., 2002, "A BEM for the Prediction of Free Surface Effects on Cavitating Hydrofoils," Comput. Mech., 28(3-4), pp. 260-274.

[27] Bal, S., 2007, "High-Speed Submerged and Surface Piercing Cavitating Hydrofoils, Including Tandem Case," Ocean Eng., 34(14), pp. 1935-1946.

[28] Bal, S., 2011, "The Effect of Finite Depth on 2D and 3D Cavitating Hydrofoils," J. Mar. Sci. Technol., 16(2), pp. 129-142.

[29] Wang, Y., Wu, X., Huang, C., and Wu, X., 2016, "Unsteady Characteristics of Cloud Cavitating Flow Near the Free Surface Around an Axisymmetric Projectile," Int. J. Multiphase Flow, 85, pp. 48-56.

[30] Gnanaskandan, A., and Mahesh, K., 2015, "A Numerical Method to Simulate Turbulent Cavitating Flows,” Int. J. Multiphase Flow, 70, pp. 22-34.

[31] Gnanaskandan, A., and Mahesh, K., 2016, "Large Eddy Simulation of the Transition From Sheet to Cloud Cavitation Over a Wedge," Int. J. Multiphase Flow, 83, pp. 86-102.

[32] Harwood, C., Young, Y., and Ceccio, S., 2016, "Ventilated Cavities on a Surface-Piercing Hydrofoil at Moderate Froude Numbers: Cavity Formation, Elimination, and Stability," J. Fluid Mech., 800, pp. 5-56.

[33] Young, Y. L., Harwood, C. M., Miguel Montero, F., Ward, J. C., and Ceccio, S. L., 2017, "Ventilation of Lifting Bodies: Review of the Physics and Discussion of Scaling Relations," ASME Appl. Mech. Rev., 69(1), p. 010801.

[34] Li, K., Wang, A., Mao, L., and Fu, S., 2013, "Shapes and Memory Effects of Natural Supercavitation of Navigating Bodies With Variable Speeds," J. Huazhong Univ. Sci. Technol. (Nat. Sci. Ed.), 41(6), p. 019.

[35] Shin, B. R., Iga, Y., and Ikohagi, T., 2001, "Numerical Analysis of Cavitating Flow Through a 2-D Decelerating Cascade," Computational Fluid Dynamics 2000 , Springer, Berlin, pp. 651-656.
[36] Chen, Y., Lu, C., Chen, X., and Cao, J., 2015, "Numerical Investigation on the Cavitation Collapse Regime Around the Submerged Vehicles Navigating With Deceleration,” Eur. J. Mech. B: Fluids, 49, pp. 153-170.

[37] Wang, Y., Huang, C., Fang, X., Wu, X., and Du, T., 2016, "On the Internal Collapse Phenomenon at the Closure of Cavitation Bubbles in a Deceleration Process of Underwater Vertical Launching," Appl. Ocean Res., 56, pp. 157-165.

[38] Zwart, P., Gerber, A., and Belarmri, T., 2004, "A Two-Phase Flow Model for Predicting Cavitation Dynamics," Fifth International Conference on MultiPhase Flow (ICMF), Yokohama, Japan, May 30-June 4.

[39] Berger, M. J., Aftosmis, M. J., and Allmaras, S., 2012, "Progress Towards a Cartesian Cut-Cell Method for Viscous Compressible Flow," AIAA Paper No. 1301.

[40] Günther, C., Hartmann, D., Schneiders, L., Meinke, M., and Schröder, W. 2011, "A Cartesian Cut-Cell Method for Sharp Moving Boundaries," AIAA Paper No. 3387.

[41] Hunt, J. C., Wray, A. A., and Moin, P., 1988, "Eddies, Streams, and Convergence Zones in Turbulent Flows," Center for Turbulence Research, Stanford University, Stanford, CA, Report No. N89-24555.

[42] Ji, B., Luo, X., Peng, X., and Wu, Y., 2013, "Three-Dimensional Large Eddy Simulation and Vorticity Analysis of Unsteady Cavitating Flow Around a Twisted Hydrofoil," J. Hydrodyn., Ser. B, 25(4), pp. 510-519.

[43] Ji, B., Luo, X., Arndt, R. E., and Wu, Y., 2014, "Numerical Simulation of Three Dimensional Cavitation Shedding Dynamics With Special Emphasis on Cavitation-Vortex Interaction," Ocean Eng., 87, pp. 64-77.

[44] Ji, B., Luo, X., Arndt, R. E., Peng, X., and Wu, Y., 2015, "Large Eddy Simulation and Theoretical Investigations of the Transient Cavitating Vortical Flow Structure Around a NACA66 Hydrofoil," Int. J. Multiphase Flow, 68, pp. 121-134. 